\title{
A fast computational formula for Kappa coefficient
}

\author{
Loc Nguyen ${ }^{1}$, Hang $\mathrm{Ho}^{2}$ \\ ${ }^{1}$ Sunflower Soft Company, Ho Chi Minh City, Vietnam \\ ${ }^{2}$ Vinh Long General Hospital, Vinh Long Province, Vietnam
}

\section{Email address:}

ng_phloc@yahoo.com (Loc Nguyen), bshangv12000@yahoo.com (Hang Ho)

\section{To cite this article:}

Loc Nguyen, Hang Ho. A Fast Computational Formula for Kappa Coefficient. Science Journal of Clinical Medicine. Vol. 4, No. 1, 2015, pp. 1-3. doi: 10.11648/j.sjcm.20150401.11

\begin{abstract}
Kappa coefficient is very important in clinical research when there is a requirement of inter-agreement among physicians who measure clinical data. It is too complicated to calculate traditional Kappa formula in huge data because of many arithmetic operations for determining probability of observed agreement and probability of chance agreement. Therefore, this research proposes a fast computational formula for Kappa coefficient based on comments about probability of observed agreement and probability of chance agreement. These comments lead to the method to save time cost when calculating Kappa coefficient and to reduce the number of arithmetic operations at least. Finally, such fast formula is applied into the gestational data measured in real world so as to evaluate its strong point.
\end{abstract}

Keywords: Kappa Coefficient, Fast Computational Formula

\section{Introduction}

Kappa coefficient is used to measure inter-agreement among persons who measure items or classify them into groups. It is very important in medical researches when the measure results on patients require the inter-agreement or assertion between physicians because such results affect mainly on clinical treatment.

Suppose two raters give their ratings to 10 items with preferences "like" or "dislike" [3], which described in table 1.

Table 1. Ratings of two users $A$ and $B$.

\begin{tabular}{llll}
\hline & & B & B \\
\cline { 3 - 4 } & & Like & Dislike \\
\hline A & Like & 4 & 1 \\
$\mathrm{~A}$ & Dislike & 1 & 4 \\
\hline
\end{tabular}

Let $\alpha$ and $\beta$ be the probability of observed agreement and the probability of chance agreement, respectively. Kappa coefficient reflects the true agreement by eliminating chance agreement from observed agreement.

$$
K=\frac{\alpha-\beta}{1-\beta}
$$

By data in above table, there are 4 items which two raters like and 4 items which they dislike. The probability of observed agreement is interpreted as the frequency of observed ratings on which both raters agree, which is calculated as below:

$$
\alpha=\frac{4+4}{10}=0.8
$$

There are 5 items per 10 items rater $A$ likes and so the chance probability that rater $A$ rates "like" is $5 / 10=0.5$. There are 5 items per 10 items rater $B$ likes and so the chance probability that rater $B$ rates "like" is $5 / 10=0.5$.

- The chance probability that both raters $A$ and $B$ rate "like" is $0.25=0.5 * 0.5$.

- The chance probability that both raters $A$ and $B$ rate "dislike" is $0.25=(1-0.5) *(1-0.5)$.

Therefore, the probability of chance agreement is the chance probability of same preference, which is the sum of above "like" and "dislike" chance probabilities [3]:

$$
\beta=0.25+0.25=0.5
$$

The Kappa coefficient is calculated as below:

$$
K=\frac{0.8-0.5}{1-0.5}=0.6
$$

There are two drawbacks of original Kappa formulae:

- The formula is very complicated and so it is impossible to get result fast when there is a requirement of fast summary about data. 
- It is easy to get wrong result in case of huge data because of the complexity.

\section{A Fast Calculation of Kappa Coefficient}

We propose a fast calculation for Kappa coefficient so as to overcome the drawback of complexity of original formula. The improvement is based on two observations:

- The probability of chance agreement $(\beta)$ doesn't exceed 0.5 [1].

- The probability of observed agreement $(\alpha)$ is inversely proportional to the probability of chance agreement with an increment number $\delta$.

Suppose that such increment number $\delta$ is the complement of chance agreement:

$$
\delta=1-\alpha
$$

Because the ratio of chance agreement $(\beta)$ doesn't exceed 0.5 , it is re-calculated as below:

$$
\beta=0.5-\delta=0.5-(1-\alpha)=\alpha-0.5
$$

Now $\beta$ is the approximate function of observed agreement. So the original Kappa formula is translated into new form so-called the fast calculation of Kappa coefficient:

$$
K=\frac{\alpha-\beta}{1-\beta}=\frac{\alpha-(\alpha-0.5)}{1-(\alpha-0.5)}=\frac{0.5}{1.5-\alpha}
$$

It is easy to recognize that the complication of traditional Kappa formula is eliminated in the new formula and what scientists do is to count the number of observed agreements Especially, computational performance is improved significantly in case of measuring inter-agreement with huge rating data. In general, the accuracy is decreased trivially. In some case that observed agreement ratio $(\alpha)$ is too small or chance agreement ration $(\beta)$ is too high (exceeds 0.5, for example) in other words; the fast calculation of Kappa produces more precise result. Back example in table 1, the Kappa coefficient is calculated according to new formula as below.

$$
K=\frac{0.5}{1.5-\alpha}=\frac{0.5}{1.5-0.8}=0.7
$$

\section{Evaluation on New Formulae}

Given gestational data [2] is composed of 2-dimension ultrasound measures of pregnant women. These women and their husbands are Vietnamese. These measures are taken at Vinh Long polyclinic - Vietnam, which include bi-parietal diameter $(b p d)$, head circumference $(h c)$, abdominal circumference $(a c)$ and fetal length $(f l)$, birth age and birth weight. These women's periods are regular and their last period is determined. Each of them has only one alive fetus. Fetal age is from 28 weeks to 42 weeks. Delivery time is not over 48 hours since ultrasound scan. Suppose that $b p d$ data requires the agreement between two physicians $A$ and $B$ who operate ultrasound scan to measure fetal $b p d$. Fetal bi-parietal diameter is measured in 40 cases and the difference of measurement between two physicians $A$ and $B$ is described in table 2. Concepts greater, equal and less indicates that data which physicians measure is greater than, equal to and less than real data. Among 40 cases, there are 24 cases that physicians agree to be equal.

Table 2: Fetal bi-parietal diameter is measured in 40 cases and the difference of measurement between two physicians $A$ and $B$.

\begin{tabular}{ccccc}
\hline \multicolumn{5}{c}{ Physician A } \\
\hline \multirow{4}{*}{ Physician B } & Greater & Equal & Less \\
& Greater & 6 & 0 & 2 \\
& Equal & 0 & 24 & 1 \\
& Less & 0 & 1 & 6 \\
\hline
\end{tabular}

By using origin Kappa formula, we have

$$
\begin{gathered}
\alpha=\frac{6+24+6}{40}=0.9 \\
\beta=\frac{8 * 6}{40^{2}}+\frac{25 * 25}{40^{2}}+\frac{7 * 9}{40^{2}}=0.430625 \\
K=\frac{0.9-0.430625}{1-0.430625}=0.8244
\end{gathered}
$$

It is easy to recognize that the calculation is very complicated and the time cost is very high. Now applying the fast formula, what we do is to look up the diagonal of above table so as to count the number of observed agreements, $6+24+6=36$, exactly. So Kappa coefficient is:

$$
K=\frac{0.5}{1.5-36 / 40}=0.83 \approx 0.8244
$$

The result is approximate to one from original formulae. In general, the proposed fast formula simplifies the traditional formula of Kappa coefficient by keeping the probability of chance agreement in average value. It means that the closer to 0.5 the probability of chance agreement is, the more precise the fast formula is. In other words, the fast formula does not always produce accurate result in all cases. Therefore, the Kappa coefficient resulted from the fast formula is reference value that help physicians to estimate the inter-agreement and so, they should re-calculate Kappa coefficient according to traditional formula if there is advanced requirement of precise Kappa coefficient in serious situation.

\section{Conclusion}

Absolutely, the fast formula is easier to be evaluated than origin formula because it eliminates complicated arithmetic operations and so what we do is to count the number of observed agreements. The basic idea of fast formula is based on two comments: (1) the ratio of chance agreement does not exceed 0.5 and (2) the probability of observed 
agreement is inversely proportional to the probability of chance agreement with a concrete deviation. The drawback of fast formula is that proportional deviation is calculated based on assumption that it is the complement of chance agreement. This assumption is heuristic assumption and so the result of fast formula is approximate to the result from original formulae. However the new formula is better than the origin formula in some cases when the probability of chance agreement never exceeds 0.5 [1] in real situation but the origin formula does not consider this situation.

\section{References}

[1] Gwet, K. (2002) Kappa Statistic is not Satisfactory for Assessing the Extent of Agreement Between Raters. Statistical Methods For Inter-Rater Reliability Assessment, No. 1, April 2002.

[2] Ho, H., Phan, D. (2011). Estimation of fetal weight from 37 to 42 weeks based on 2-dimensional ultrasound measures. Journal of Practical Medicine, volume 12 (797) 2011, pp. 89.

[3] Wikipedia. (2014) Cohen's kappa. URL (last checked September 13 2014) http://en.wikipedia.org/wiki/Cohen\%27s_kappa. 